\title{
Regio- and Diastereoselective Vinylogous Mannich Addition of 3-Alkenyl-2-oxindoles to $\alpha$-Fluoroalkyl Aldimines
}

\author{
Yingle Liu ${ }^{\mathrm{a}, \mathrm{b}}$ \\ Yi Yang ${ }^{a}$ \\ Yangen Huang ${ }^{b}$ \\ Xiu-Hua Xuc \\ Feng-Ling Qing*b,c \\ a School of Chemistry and Pharmaceutical Engineering, Sichuan \\ University of Science \& Engineering, 180 Xueyuan Street, Huixing \\ Lu, Zigong, Sichuan 643000, P. R. of China \\ b College of Chemistry, Chemical Engineering and Biotechnology, \\ Donghua University, 2999 North Renmin Road, Shanghai \\ 201620, P. R. of China \\ ' Key Laboratory of Organofluorine Chemistry, Shanghai Institute \\ of Organic Chemistry, Chinese Academy of Sciences, 345 Lingling \\ Road, Shanghai 200032, P. R. of China \\ fla@mail.sioc.ac.cn
}
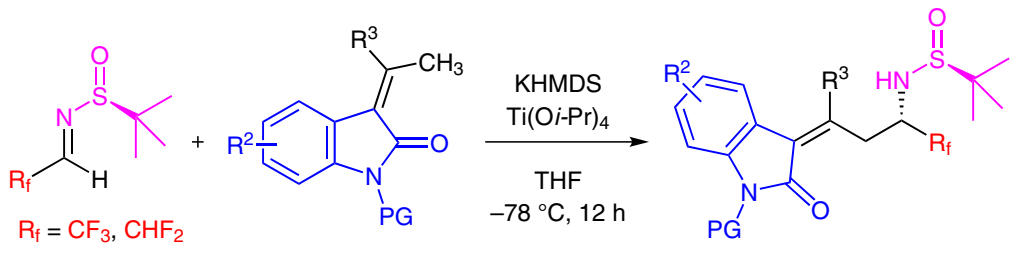

14 examples, $55-92 \%$ yield up to $98: 2 \mathrm{dr},>99: 1 \mathrm{ZIE}$
Received: 24.09.2014

Accepted after revision: 03.11.2014

Published online: 20.11 .2014

DOI: 10.1055/s-0034-1379600; Art ID: st-2014-w0796-c

Abstract An efficient asymmetric vinylogous Mannich (AVM) addition reaction of 3-alkenyl-2-oxindoles to $\alpha$-fluoroalkyl aldimines has been developed. This reaction provided various optical active $\alpha$-alkylidene$\delta$-amino- $\delta$-fluoroalkyl oxindoles in excellent yields, complete $\gamma$-site regioselectivity, and excellent diastereoselectivities.

Key words fluoroalkylated compounds, oxindole, asymmetric, Mannich addition

The $\delta$-amino- $\alpha, \beta$-unsaturated carbonyl compounds represent an important class of units in modern organic and medicinal chemistry. ${ }^{1}$ They are useful building blocks for the synthesis of various pharmaceuticals and biologically active natural products. ${ }^{2}$ It is well known that fluorinecontaining compounds are considered as the extraordinarily promising drug candidates because the introduction of fluorine atom or fluorine-containing groups into organic compounds often significantly improves the chemical, physical, and biological properties of the parent compound. ${ }^{3,4}$ Especially, the fluoroalkyl-substituted molecules, such as trifluoromethylated and difluoromethylated compounds, have attracted increasing attention. ${ }^{5}$ Thus, the incorporation of fluoroalkyl into $\delta$-amino- $\alpha, \beta$-unsaturated carbonyl compounds will provide novel fluorinated moieties, which might be applied in various research fields. Among them, $\delta$-amino- $\delta$-fluoroalkyl- $\alpha, \beta$-unsaturated carbonyl compounds are particularly interesting, because the neighboring electron-withdrawing fluoroalkyl groups would change the basicity of imine groups, thus affecting their bioactivities. Normally, these compounds were prepared by vinylogous Mannich reactions. ${ }^{6}$ In 1992, Tsukamoto and Kitazume reported the Lewis acid promoted reaction of fluorinated $\mathrm{N}, \mathrm{O}$-acetal with trimethylsilyloxyfuran
(Scheme 1, a). ${ }^{7}$ The Lewis acid catalyzed vinylogous Mannich addition of trimethylsilyloxyfuran to fluorinated aldimines was disclosed by Crousse and co-workers in 2004 (Scheme 1, a). ${ }^{8}$ Shi's group realized the first enantioselective vinylogous Mannich reaction of fluorinated aldimines bearing a chiral auxiliary [(S)-1-phenylethyl group] and siloxyfurans under the catalytic environment of silver acetate and axially chiral phosphine-oxazoline ligand (Scheme 1, b). ${ }^{9}$ Very recently, we developed a tunable and highly regioand diastereoselective addition reaction of acyclic silyl dienolates to $\alpha$-fluoroalkyl sulfinylimines, in which the Lewis acid TMSOTf was a critical parameter in the control of $\gamma$-site regioselectivity (Scheme 1, c). ${ }^{10}$ All the previous works need silylated substrates as the nucleophiles. From the point of atom and step economy, it is worthy to investigate the addition reactions directly using $\alpha, \beta$-unsaturated carbonyl compounds as the nucleophiles. In light of the important pharmaceutical implications of the privileged structural motif oxindole, ${ }^{11}$ herein we report a regio- and diastereoselective vinylogous Mannich addition of 3-alkenyl-2-oxindoles to $\alpha$ fluoroalkyl aldimines to afford various chiral $\alpha$-alkylidene$\delta$-amino- $\delta$-fluoroalkyl oxindoles (Scheme 1, d).

Initially, the reaction conditions were optimized using $\left(S_{S}\right)$-N-tert-butanesulfinyl-3,3,3-trifluoroacetaldimine $(\mathbf{1 a})^{12}$ and $\mathrm{N}$-Boc-protected 3 -alkylidene-2-oxindole $\mathbf{2} \mathbf{a}^{13}$ as the model substrates (Table 1 ). Treatment of the substrates with TMSOTf and $\mathrm{Et}_{3} \mathrm{~N}$ gave only a silylated intermediate of $\mathbf{2 a} .^{13 \mathrm{~d}}$ The desired product 3a was not obtained (Table 1, entry 1 ). In view of the better nucleophilic properties of the metallic enolate intermediate in comparison to silyl enolate, LDA was chosen as the base. To our delight, the addition reaction happened in the presence of LDA, and $\mathbf{3 a}$ was obtained in moderate yield, with virtually complete $\gamma$-site selectivity $(>99: 1 \mathrm{\gamma} / \alpha)$ and good diastereoselectivity $(94: 6 \mathrm{dr}, Z / E=$ $8: 1$; Table 1 , entry 2 ). Considering the fact that the addition of Lewis acid might improve the yield and diastereoselec- 

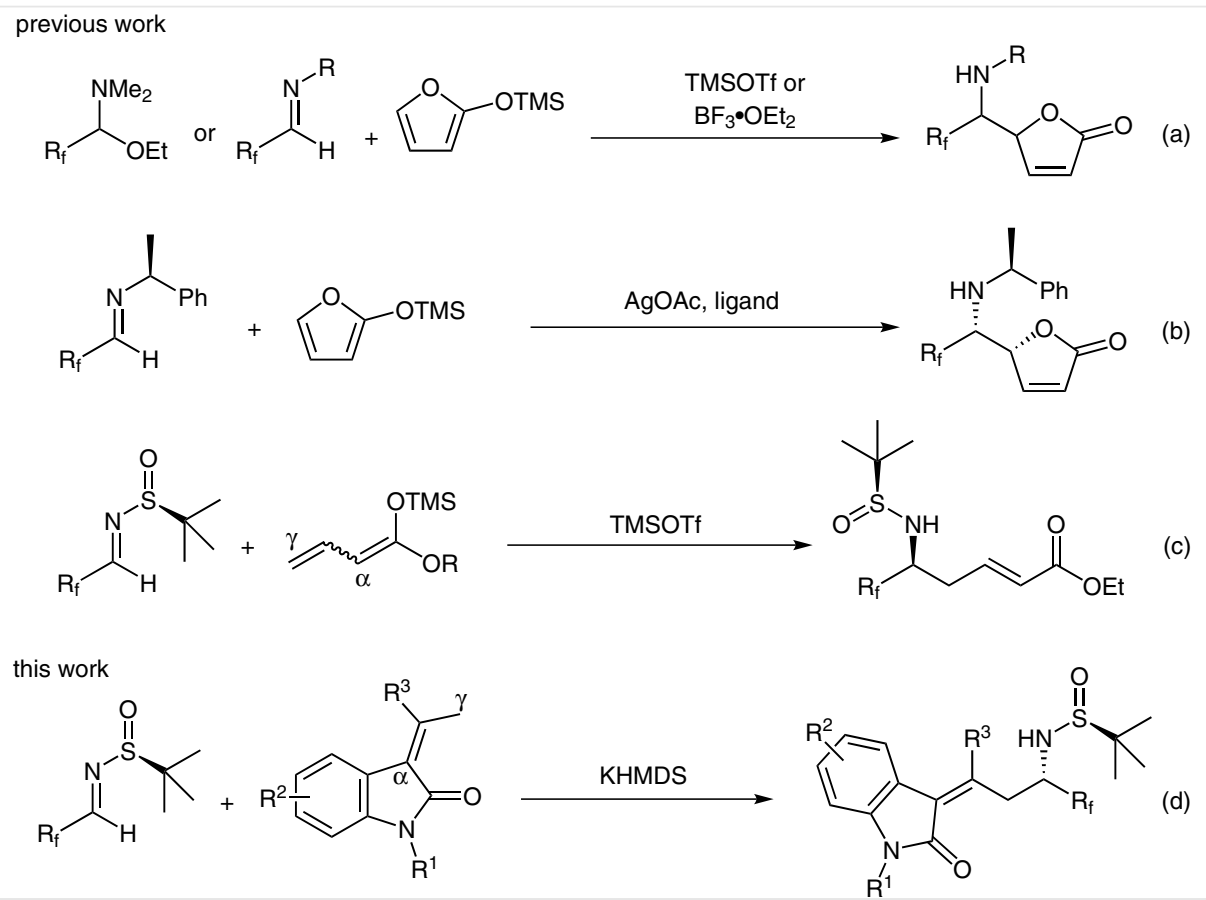

Scheme 1 Synthesis of $\delta$-amino- $\alpha, \beta$-unsaturated carbonyl compounds by vinylogous Mannich reactions

tivity because of its coordination with sulfinylimine substrate, ${ }^{14}$ different Lewis acids were then investigated. Among the three typical Lewis acids, $\mathrm{Ti}(\mathrm{Oi}-\mathrm{Pr})_{4}, \mathrm{AlMe}_{3}$, and $\mathrm{BF}_{3} \cdot \mathrm{OEt}_{2}, \mathrm{Ti}(\mathrm{O} i \text {-Pr })_{4}$ showed the highest efficiency and sharply increased the yield of 3a from $60 \%$ to $98 \%$ (Table 1 , entries 3-5). When the base was changed from LDA to KHMDS, 3a was obtained in similar yield with much higher $Z / E$ ratio (Table 1 , entry 6$)$. Finally, different solvents including toluene, $\mathrm{Et}_{2} \mathrm{O}$, and hexane were screened (Table 1, entries 7-9). However, no better result was obtained.

With the optimized conditions in hand, the substrate scope of direct asymmetric vinylogous Mannich (AVM) reaction was surveyed. ${ }^{15,16}$ The results are summarized in Scheme 2. Firstly, 3-alkylidene-2-oxindoles 2a-d bearing diverse nitrogen protecting groups, Boc, Moc, Bn, and Me, reacted smoothly with 1a under identical conditions, affording the corresponding products $\mathbf{3 a - d}$ in moderate to good yields and excellent stereoselectivities. Additionally, the reaction conditions displayed good compatibility with the substituent pattern on the phenyl ring of the 2-oxindole. The substrates $\mathbf{2 e - g}$, bearing electron-donating and electron-withdrawing groups, can be efficiently transformed to the corresponding products in excellent yields and stereoselectivities. Subsequently, the patterns of $\mathrm{R}^{3}$ in 3-alkylidene-2-oxindole $\mathbf{2} \mathbf{h}$-j having aromatic groups were tested as the substrates. The reactions proceeded well affording products $\mathbf{3 h}-\mathbf{j}$ in good yields and diastereoselectivities, although the $Z / E$ ratios were comparably low. It was noteworthy that this protocol could be applied to difluoromethylated sulfinylimine $\mathbf{1 b}$. The corresponding difluoromethylated products $\mathbf{3 k}-\mathbf{n}$ were conveniently obtained under the optimal reaction conditions. The 3-(propan2-ylidene)benzofuran-2(3H)-one $\mathbf{2 0}^{17}$ was also a suitable substrate for this reaction to furnish the product $\mathbf{3 o}$ in modest yield and good stereoselectivity.

The absolute configuration of these $\alpha$-alkylidene- $\delta$ amino- $\delta$-fluoroalkyl oxindoles 3 was confirmed by X-ray crystallographic analysis of compounds 3d (Figure 1). ${ }^{18}$ Normally, a nonchelated transition-state model was involved in the addition reaction of nucleophiles to fluorinated sulfinylimines. ${ }^{12}$ The stereochemical outcome observed in the present study could also be explained by the nonchelated transition-state model, in which the sulfinyl oxygen coordinates to $\mathrm{Ti}(\mathrm{O} i-\mathrm{Pr})_{4}$ and sterically shields the Re face of the imine. Thus, the $\mathrm{Si}$ attack from metallic enolate intermediates would produce adducts 3 with $\left(C_{S}, S_{S}\right)$-configurations. The high $Z / E$ ratios in compound 3 might be caused by the cyclic structure of nucleophilic enolate intermediates. ${ }^{19}$ The accurate reaction mechanism still needs further investigation. 


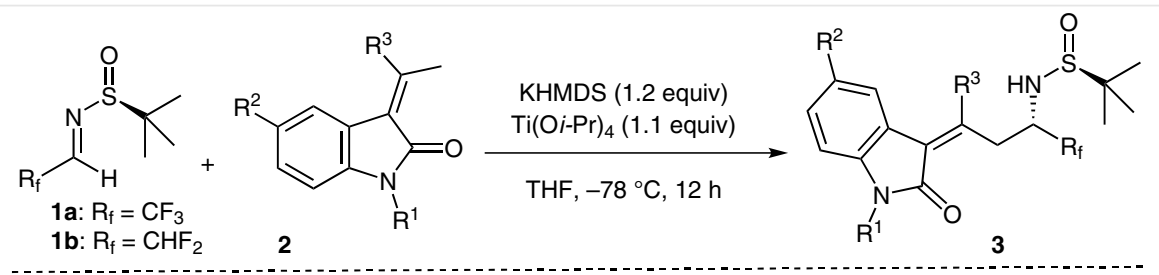<smiles>C/C(C[C@H](NS(=O)C(C)(C)C)C(F)(F)F)=C1\C(=O)N(C(C)(C)C)c2ccccc21</smiles>

3a, $89 \%$ yield 93:7 dr, 94:6 ZIE<smiles>C/C(C[C@H](NS(=O)C(C)(C)C)C(F)(F)F)=C1/C(=O)N(C)c2ccccc21</smiles>

3d, $80 \%$ yield 93:7 dr, 94:6 ZIE<smiles>C/C(C[C@H](NS(=O)C(C)(C)C)C(F)(F)F)=C1/C(=O)N(C(C)(C)C)c2ccc(Cl)cc21</smiles>

$3 \mathbf{g}, 91 \%$ yield 95:5 dr, 95:5 ZIE

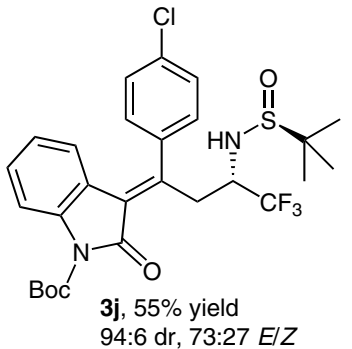<smiles>COc1ccc2c(c1)/C(=C(\C)C[C@H](CF)NS(=O)C(C)(C)C)C(=O)N2C(=O)OC(C)(C)C</smiles>
$3 m, 78 \%$ yield 93:7 dr, 91:9 ZIE<smiles>C/C(C[C@H](NS(=O)C(C)(C)C)C(F)(F)F)=C1\C(=O)N(C)c2ccccc21</smiles>

3 b, $62 \%$ yield 90:10 dr, 99:1 ZIE<smiles>C/C(C[C@H](NS(=O)C(C)(C)C)C(F)(F)F)=C1\C(=O)N(C(C)(C)C)c2ccc(C)cc21</smiles>

3e, $92 \%$ yield

94:6 dr, 91:9 ZIE

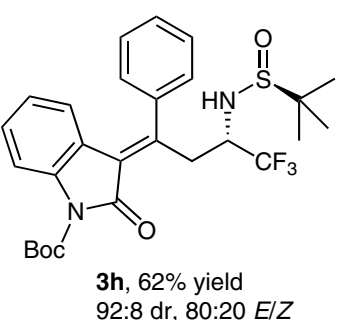<smiles>C/C(C[C@H](NS(=O)C(C)(C)C)C(F)(F)F)=C1\C(=O)N(Cc2ccccc2)c2ccccc21</smiles>

Scheme 2 Vinylogous Mannich addition of 3-alkenyl-2-oxindoles to $\alpha$-fluoroalkyl aldimines 
Table 1 Optimization of Reaction Conditions ${ }^{\mathrm{a}}$<smiles>[Y]C([3H])=C1C(=O)N(C(=O)OC(C)(C)C)c2ccccc21</smiles><smiles>C/C(C[C@H](NS(=O)C(C)(C)C)C(F)(F)F)=C1\C(=O)N(C(C)(C)C)c2ccccc21</smiles>

2a

\begin{tabular}{|c|c|c|c|c|c|c|c|}
\hline Entry & Base & Lewis acid & Solvent & $\begin{array}{l}\text { Temp } \\
\left({ }^{\circ} \mathrm{C}\right)\end{array}$ & $\begin{array}{l}\text { Yield } \\
(\%)^{\mathrm{b}}\end{array}$ & $Z \mid E^{b}$ & $d r^{b}$ \\
\hline $1^{c}$ & $\mathrm{Et}_{3} \mathrm{~N}$ & TMSOTf & $\mathrm{CH}_{2} \mathrm{Cl}_{2}$ & 0 to r.t. & 0 & - & \\
\hline 2 & LDA & - & THF & -78 & 60 & $8: 1$ & $94: 6$ \\
\hline 3 & LDA & $\mathrm{Ti}(\mathrm{Oi}-\mathrm{Pr})_{4}$ & THF & -78 & 98 & $6: 1$ & $94: 6$ \\
\hline 4 & LDA & $\mathrm{AlMe}_{3}$ & THF & -78 & 58 & $12: 1$ & $92: 8$ \\
\hline 5 & LDA & $\mathrm{BF}_{3} \cdot \mathrm{OEt}_{2}$ & THF & -78 & 70 & 7:1 & $95: 5$ \\
\hline 6 & KHMDS & $\mathrm{Ti}(\mathrm{Oi}-\mathrm{Pr})_{4}$ & THF & -78 & 97 & $16: 1$ & $93: 7$ \\
\hline 7 & KHMDS & $\mathrm{Ti}(\mathrm{Oi}-\mathrm{Pr})_{4}$ & toluene & -78 & 87 & $12: 1$ & $93: 7$ \\
\hline 8 & KHMDS & $\mathrm{Ti}(\mathrm{Oi}-\mathrm{Pr})_{4}$ & $\mathrm{Et}_{2} \mathrm{O}$ & -78 & 41 & $2: 1$ & $94: 6$ \\
\hline 9 & KHMDS & $\mathrm{Ti}(\mathrm{Oi}-\mathrm{Pr})_{4}$ & hexane & -78 & 18 & - & \\
\hline
\end{tabular}

${ }^{a}$ Reactions were carried out using $\mathbf{1 a}(0.3 \mathrm{mmol}), \mathbf{2 a}(0.36 \mathrm{mmol}, 1.2$ equiv), base ( $0.36 \mathrm{mmol}, 1.2$ equiv), and Lewis acid ( $0.33 \mathrm{mmol}, 1.1$ equiv) in dry solvent $(2.5 \mathrm{~mL})$ for $12 \mathrm{~h}$.

${ }^{\mathrm{b}}$ Ratios and yields were determined by ${ }^{19} \mathrm{~F}$ NMR spectroscopy of the crude reaction mixture using benzotrifluoride as an internal standard.

' Base (0.33 mmol, 1.1 equiv) and Lewis acid ( $0.36 \mathrm{mmol}, 1.2$ equiv).

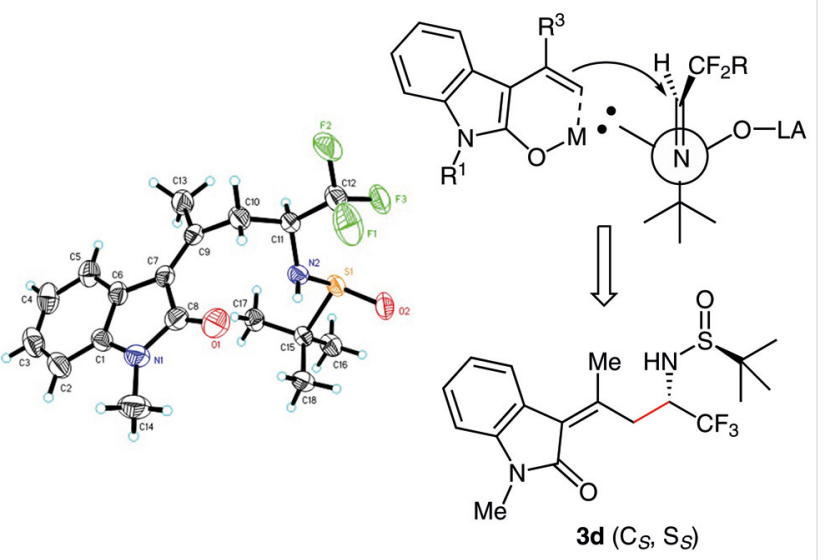

Figure 1 X-ray crystal structure of $\mathbf{3 d}$ and proposed transition-state model

It should be mentioned that the $N$-tert-butylsulfinyl group can serve not only as an efficient chiral auxiliary, but also as an amine protecting group. ${ }^{20}$ It could be readily cleaved under mild acidic conditions. After deprotection, the trifluoromethylated free amines $\mathbf{4}$ can be easily obtained in high yield (Scheme 3).

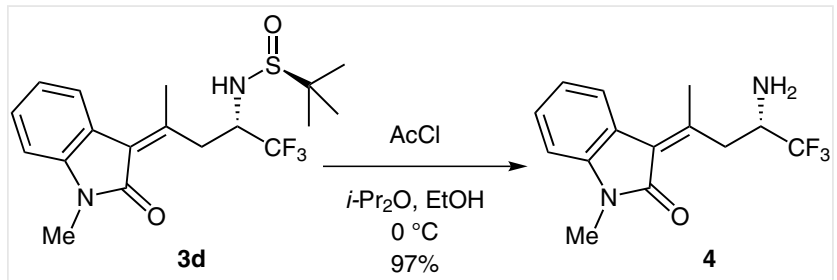

Scheme 3 Conversion of $\mathbf{3 d}$ into the free primary amine $\mathbf{4}$

In summary, we have demonstrated a practical and efficient approach to synthesize $\alpha$-alkylidene- $\delta$-amino- $\delta$-fluoroalkyl oxindoles via a regio- and stereoselective vinylogous Mannich-type reaction of fluorinated $N$-tert-butanesulfinyl aldimines with 3-alkenyl-2-oxindoles. This protocol displayed broad substrate scope, good functional-group compatibility, and satisfactory stereocontrol. Further applications of this method for the preparation of new fluorinated bioactive molecules are in progress.

\section{Acknowledgment}

We thank the National Natural Science Foundation of China $(21421002,21332010,21272036)$ and the National Basic Research Program of China (2012CB21600) for financial support. Sichuan University of Science \& Engineering (No. 2014RC07, 2012RC17), Zigong Science and Technology Bureau (No. 2013X02), Education Department of Sichuan Province (No. 14ZB0207) and Key Laboratory of Green Chemistry of Sichuan Institutes of Higher Education (No. LZJ1401) are also gratefully acknowledged for funding this work.

\section{Supporting Information}

Supporting information for this article is available online at http://dx.doi.org/10.1055/s-0034-1379600.

\section{References and Notes}

(1) For selected reviews, see: (a) Casiraghi, G.; Zanardi, F.; Appendino, G.; Rassu, G. Chem. Rev. 2000, 100, 1929. (b) Casiraghi, G.; Zanardi, F.; Battistini, L.; Rassu, G. Synlett 2009, 1525. (c) Casiraghi, G.; Battistini, L.; Curti, C.; Rassu, G.; Zanardi, F. Chem. Rev. 2011, 111, 3076. (d) Martin, S. F. Adv. Heterocycl. Chem. 2013, 110, 73.

(2) For selected examples, see: (a) Mohan, S.; Kerry, P. S.; Bance, N.; Niikura, M.; Pinto, B. M. Angew. Chem. Int. Ed. 2014, 53, 1076. (b) Ren, W.; Wang, Q.; Zhu, J. Angew. Chem. Int. Ed. 2014, 53, 1818. (c) Hickin, J. A.; Ahmed, A.; Fucke, K.; Ashcroft, M.; Jones, K. Chem. Commun. 2014, 50, 1238. (d) Abels, F.; Lindemann, C.; Schneider, C. Chem. Eur. J. 2014, 20, 1964. (e) Sartori, A.; Dell'Amico, L.; Battistini, L.; Curti, C.; Rivara, S.; Pala, D.; Kerry, 
P. S.; Pelosi, G.; Casiraghi, G.; Rassu, G.; Zanardi, F. Org. Biomol. Chem. 2014, 12, 1561. (f) Suetsugu, S.; Nishiguchi, H.; Tsukano, C.; Takemoto, Y. Org. Lett. 2014, 16, 996.

(3) (a) Kirsch, P. Modern Fluoroorganic Chemistry; Wiley-VCH: Weinheim, 2004. (b) Uneyama, K. Organofluorine Chemistry; Blackwell: Oxford, 2006. (c) Bégué, J.-P.; Bonnet-Delpon, D. Bioorganic and Medicinal Chemistry of Fluorine; John Wiley and Sons: Hoboken, 2008. (d) Ojima, I. Fluorine in Medicinal Chemistry and Chemical Biology; Wiley-Blackwell: Oxford, 2009. (e) Gouverneur, V.; Müler, K. Fluorine in Pharmaceutical and Medicinal Chemistry: From Biophysical Aspects to Clinical Applications; Imperial College Press: London, 2012.

(4) (a) Müller, K.; Faeh, C.; Diederich, F. Science 2007, 317, 1881. (b) Purser, S.; Moore, P. R.; Swallow, S.; Gouverneur, V. Chem. Soc. Rev. 2008, 37, 320. (c) Meanwell, N. A. J. Med. Chem. 2011, 54, 2529. (d) Wang, J.; Sánchez-Roselló, M.; Aceña, J. L.; Pozo, C.; Sorochinsky, A. E.; Fustero, S.; Soloshonok, V. A.; Liu, H. Chem. Rev. 2014, 114, 2432.

(5) For selected reviews, see: (a) Nie, J.; Guo, H.-C.; Cahard, D.; Ma, J.-A. Chem. Rev. 2011, 111, 455. (b) Tomashenko, O. A.; Grushin, V. V. Chem. Rev. 2011, 111, 4475. (c) Furuya, T.; Kamlet, A. S.; Ritter, T. Nature (London, U.K.) 2011, 473, 470. (d) Studer, A. Angew. Chem. Int. Ed. 2012, 51, 8950. (e) Liang, T.; Neumann, C. N.; Ritter, T. Angew. Chem. Int. Ed. 2013, 52, 8214. (f) Merino, E.; Nevado, C. Chem. Soc. Rev. 2014, 43, 6598.

(6) (a) Bur, S. K.; Martin, S. F. Tetrahedron 2001, 57, 3221. (b) Martin, S. F. Acc. Chem. Res. 2002, 35, 895. (c) Karimi, B.; Enders, D.; Jafari, E. Synthesis 2013, 45, 2769.

(7) Tsukamoto, T.; Kitazume, T. Chem. Lett. 1992, 21, 1377.

(8) Spanedda, M. V.; Ourévitch, M.; Crousse, B.; Bégué, J.-P.; Bonnet-Delpon, D. Tetrahedron Lett. 2004, 45, 5023.

(9) (a) Zhao, Q.-Y.; Yuan, Z.-L.; Shi, M. Tetrahedron: Asymmetry 2010, 21, 943. (b) Zhao, Q.-Y.; Yuan, Z.-L.; Shi, M. Adv. Synth. Catal. 2011, 353, 637.

(10) Liu, Y.; Liu, J.; Huang, Y.; Qing, F.-L. Chem. Commun. 2013, 49, 7492.

(11) For selected reviews, see: (a) Galliford, C. V.; Scheidt, K. A. Angew. Chem. Int. Ed. 2007, 46, 8748. (b) Peddibhotla, S. Curr. Bioact. Compd. 2009, 5, 20. (c) Zhou, F.; Liu, Y.-L.; Zhou, J. Adv. Synth. Catal. 2010, 352, 1381.

(12) (a) Truong, V. L.; Ménard, M. S.; Dion, I. Org. Lett. 2007, 9, 683. (b) Truong, V. L.; Pfeiffer, J. Y. Tetrahedron Lett. 2009, 50, 1633. (c) Mei, H.; Xiong, Y.; Han, J.; Pan, Y. Org. Biomol. Chem. 2011, 9, 1402. (d) Zhang, H.; Li, Y.; Xu, W.; Zheng, W.; Zhou, P.; Sun, Z. Org. Biomol. Chem. 2011, 9, 6502. (e) Shibata, N.; Nishimine, T.; Shibata, N.; Tokunaga, E.; Kawada, K.; Kagawa, T.; Sorochinskycde, A. E.; Soloshonok, V. A. Chem. Commun. 2012, 48, 4124. (f) Turcheniuk, K. V.; Poliashko, K. O.; Kukhar, V. P.; Rozhenko, A. B.; Soloshonok, V. A.; Sorochinsky, A. E. Chem. Commun. 2012, 48, 11519. (g) Röschenthaler, G.-V.; Kukhar, V. P.; Kulik, I. B.; Belik, M. Y.; Sorochinsky, A. E.; Rusanov, E. B.; Soloshonok, V. A. Tetrahedron Lett. 2012, 53, 539. (h) Mei, H.; Xie, C.; Wu, L.; Soloshonok, V. A.; Han, J.; Pan, Y. Org. Biomol. Chem. 2013, 11, 8018. (i) Shevchuk, M. V.; Kukhar, V. P.; Röschenthaler, G.-V.; Bassil, B. S.; Kawada, K.; Soloshonok, V. A.; Sorochinsky, A. E. RSC Adv. 2013, 3, 6479. (j) Xie, C.; Mei, H.; Wu, L.; Soloshonok, V. A.; Han, J.; Pan, Y. Eur. J. Org. Chem. 2014, 1445. (k) Mei, H.; Dai, Y.; Wu, L.; Soloshonok, V. A.; Han, J.; Pan, Y. Eur. J. Org. Chem. 2014, 2429. (l) Milcent, T.; Hao, J.; Kawada, K.; Soloshonok, V. A.; Ongeri, S.; Crousse, B. Eur. J. Org. Chem.
2014, 3072. (m) Xie, C.; Mei, H.; Wu, L.; Han, J.; Soloshonok, V. A.; Pan, Y. J. Fluorine Chem. 2014, 165, 67. (n) Wu, L.; Xie, C.; Mei, H.; Soloshonok, V. A.; Han, J.; Pan, Y.J. Org. Chem. 2014, 79, 7677. (o) Shibata, N.; Nishimine, T.; Shibata, N.; Tokunaga, E.; Kawada, K.; Kagawa, T.; Aceña, J. L.; Sorochinsky, A. E.; Soloshonok, V. A. Org. Biomol. Chem. 2014, 12, 1454. (p) Mei, H.; Xiong, Y.; Xie, C.; Soloshonok, V. A.; Han, J.; Pan, Y. Org. Biomol. Chem. 2014, 12, 2018. (q) Wu, L.; Xie, C.; Mei, H.; Soloshonok, V. A.; Han, J.; Pan, Y. Org. Biomol. Chem. 2014, 12, 4620. (r) Xie, C.; Wu, L.; Mei, H.; Soloshonok, V. A.; Han, J.; Pan, Y. Org. Biomol. Chem. 2014, 12, 7836. (s) Qian, P.; Xie, C.; Wu, L.; Mei, H.; Soloshonok, V. A.; Han, J.; Pan, Y. Org. Biomol. Chem. 2014, 12, 7909. (t) Xie, C.; Mei, H.; Wu, L.; Soloshonok, V. A.; Han, J.; Pan, Y. RSC Adv. 2014, 4, 4763. (u) Xie, C.; Wu, L.; Mei, H.; Soloshonok, V. A.; Han, J.; Pan, Y. Tetrahedron Lett. 2014, 55, 5908.

(13) (a) Curti, C.; Rassu, G.; Zambrano, V.; Pinna, L.; Pelosi, G.; Sartori, A.; Battistini, L.; Zanardi, F.; Casiraghi, G. Angew. Chem. Int. Ed. 2012, 51, 6200. (b) Rassu, G.; Zambrano, V.; Tanca, R.; Sartori, A.; Battistini, L.; Zanardi, F.; Curti, C.; Casiraghi, G. Eur. J. Org. Chem. 2012, 466. (c) Rassu, G.; Zambrano, V.; Pinna, L.; Curti, C.; Battistini, L.; Sartori, A.; Pelosi, G.; Zanardi, F.; Casiraghi, G. Adv. Synth. Catal. 2013, 355, 1881. (d) Ranieri, B.; Sartori, A.; Curti, C.; Battistini, L.; Rassu, G.; Pelosi, G.; Casiraghi, G.; Zanardi, F. Org. Lett. 2014, 16, 932. (e) Chen, Q.; Wang, G.; Jiang, X.; Xu, Z.; Lin, L.; Wang, R. Org. Lett. 2014, 16, 1394.

(14) (a) Jiang, W.; Chen, C.; Marinkovic, D.; Tran, J. A.; Chen, C. W.; Arellano, L. M.; White, N. S.; Tucci, F. C. J. Org. Chem. 2005, 70, 8924. (b) Senanayake, C.; Lu, B. Z.; Li, N.; Han, Z.; Bakale, R. P.; Wald, S. A. Org. Lett. 2005, 7, 2599. (c) Larhed, M.; Arefalk, A.; Wannberg, J.; Hallberg, A. J. Org. Chem. 2006, 71, 1265.

(15) General Procedure for the Synthesis of $\alpha$-Alkylidene-

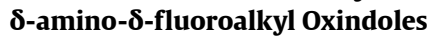

A solution of KHMDS ( $0.36 \mathrm{~mL}, 1 \mathrm{M}$ solution in THF) was slowly added to a dried Scheck flask containing 3-alkenyl-2-oxindoles $2(0.36 \mathrm{mmol})$ in THF $(2.0 \mathrm{~mL})$ at $-78^{\circ} \mathrm{C}$ under $\mathrm{N}_{2}$ atmosphere. After stirring at $-78{ }^{\circ} \mathrm{C}$ for $1 \mathrm{~h}$, the mixture of $\mathbf{1}(0.3 \mathrm{mmol})$ and $\mathrm{Ti}(\mathrm{O} i-\mathrm{Pr})_{4}(0.33 \mathrm{mmol})$ in THF $(1.0 \mathrm{~mL})$ was added dropwise, and the mixture was stirred for $12 \mathrm{~h}$ at $-78^{\circ} \mathrm{C}$. Then sat. aq $\mathrm{NH}_{4} \mathrm{Cl}$ solution and $\mathrm{H}_{2} \mathrm{O}$ was added at $-78^{\circ} \mathrm{C}$. The mixture was brought to r.t. After $5 \mathrm{~min}$, the mixture was filtered through Celite, and the filtrate was extracted with EtOAc. The combined organic solution was dried over $\mathrm{MgSO}_{4}$. After the removal of volatile solvents under vacuum, the crude product was purified by silica gel column chromatography to give the required product.

(16) Analytical Data for Compound 3a Mp 60-61 ${ }^{\circ} \mathrm{C} .[\alpha]_{\mathrm{D}}{ }^{17.0}+187.7\left(\mathrm{c} 0.41, \mathrm{CHCl}_{3}\right) .{ }^{1} \mathrm{H}$ NMR $(400 \mathrm{MHz}$, $\left.\mathrm{CDCl}_{3}\right): \delta=7.88(\mathrm{~d}, J=8.2 \mathrm{~Hz}, 1 \mathrm{H}), 7.60(\mathrm{~d}, J=7.8 \mathrm{~Hz}, 1 \mathrm{H}), 7.35$ $(\mathrm{t}, J=7.9 \mathrm{~Hz}, 1 \mathrm{H}), 7.20(\mathrm{t}, J=7.7 \mathrm{~Hz}, 1 \mathrm{H}), 4.35(\mathrm{dd}, J=24.4,11.2$ $\mathrm{Hz}, 2 \mathrm{H}), 4.19-3.96$ (m, $1 \mathrm{H}), 2.62(\mathrm{dd}, J=12.7,3.8 \mathrm{~Hz}, 1 \mathrm{H}), 2.43$ (s, $3 \mathrm{H}), 1.67$ (s, $9 \mathrm{H}), 1.06(\mathrm{~s}, 9 \mathrm{H}) .{ }^{19} \mathrm{~F} \mathrm{NMR}\left(377 \mathrm{MHz}, \mathrm{CDCl}_{3}\right): \delta$ $=-74.87(\mathrm{~d}, J=6.5 \mathrm{~Hz}, 3 \mathrm{~F}) \cdot{ }^{13} \mathrm{C}$ NMR $\left(101 \mathrm{MHz}, \mathrm{CDCl}_{3}\right): \delta=$ $166.5,152.0,149.1,138.3,128.9,125.6,125.2(\mathrm{q}, J=284.8 \mathrm{~Hz})$, $124.1,123.9,123.2,114.6,84.6,57.2,56.4$ (q, $J=30.2 \mathrm{~Hz}), 35.3$, 28.1, 24.2, 22.2. IR (KBr): $v_{\max }=3261,3060,2975,1731,1613$, $1462,1535,1300,1258,1157,1090,841,748 \mathrm{~cm}^{-1}$. MS (EI): $\mathrm{m} / z$ $=497.2[\mathrm{M}+\mathrm{Na}]^{+}$. ESI-HRMS: $m / z[\mathrm{M}+\mathrm{Na}]^{+}$calcd for $\mathrm{C}_{22} \mathrm{H}_{29} \mathrm{~F}_{3} \mathrm{~N}_{2} \mathrm{O}_{4} \mathrm{SNa}$ : 497.1692; found: 497.1712.

(17) Trost, B. M.; Cramer, N.; Silverman, S. M. J. Am. Chem. Soc. 2007, $129,12396$. 
(18) Further details of the crystal data can be obtained from the Cambridge Crystallographic Data Centre, 12 Union Road, Cambridge, CB2 1EZ, UK (CCDC deposition No. 1011207).
(19) Vincent, M. A.; Smith, A. C.; Donnard, M.; Harford, P. J.; Haywood, J.; Hillier, I. H.; Clayden, J.; Wheatley, A. E. H. Chem. Eur. J. 2012, 18, 11036.

(20) Robak, M. T.; Herbage, M. A.; Ellman, J. A. Chem. Rev. 2010, 110, 3600 . 